\title{
Achondrogenesis type IA: clinical, histologic, molecular, and prenatal ultrasound diagnosis
}

This article was published in the following Dove Press journal:

The Application of Clinical Genetics

\author{
Sara Vanegas' \\ Luz Fernanda Sua ${ }^{2}$ \\ Jaime López-Tenorio ${ }^{3}$ \\ Diana Ramírez-Montaño' \\ Harry Pachajoa ${ }^{1,4}$ \\ 'Department of Basic Medical \\ Sciences, Center for Research on \\ Congenital Anomalies and Rare \\ Diseases (CIACER), Universidad \\ Icesi, Cali, Colombia; ${ }^{2}$ Department \\ of Pathology and Laboratory \\ Medicine, Fundación Valle del Lili, Cali, \\ Colombia; ${ }^{3}$ Department of Obstetrics \\ and Perinatology, Fundación Valle del \\ Lili, Cali, Colombia; ${ }^{4}$ Department of \\ Pediatric Medical Genetics, Fundación \\ Valle del Lili, Cali, Colombia
}

Background: Achondrogenesis type IA (ACG1A) is a rare, lethal autosomal recessive chondrodysplasia affecting endochondral bone ossification and differentiation, causing intrauterine growth restriction, narrow thorax, and short limbs. Mutations in TRIP11, which encodes Golgi microtubule-binding protein 210 in the Golgi apparatus, alter protein transport in tissues.

Case presentation: A 28-week gestation male fetus was diagnosed with ACG1A by clinical, radiological, histologic, and molecular findings.

Results: Whole exome sequencing was performed on fetal DNA and parental blood. Two fetal heterozygous novel variants of TRIP11, c.2304_2307delTCAA (p.Asn768Lysfs*7) and c.2128_2129delAT (p.1le710Cysfs*19), were inherited from the mother and father, respectively. Both variants created a reading frameshift leading to a premature stop codon and loss of protein function.

Conclusion: To our knowledge, this is the first Latin American report with clinical, radiographic, and molecular diagnosis of ACG1A. Clinical and molecular diagnosis in utero is essential for genotype-phenotype correlation and is useful for providing better genetic counseling.

Keywords: achondrogenesis type IA, endochondral bone, TRIP11, GMAP-210

\section{Introduction}

Achondrogenesis type IA (ACG1A; MIM \#200600) is a rare, lethal autosomal recessive skeletal dysplasia characterized by severe demineralization of intramembranous and endochondral bone, resulting in thoracic hypoplasia, severe micromelia, hypocalcification, and tubular bone fracture. Some studies have reported a short neck, protruding eyes, flat nasal bridge, and low-set ears. ${ }^{1,2}$ Affected individuals die in utero or shortly after birth. Radiological findings show short-cupped flared ribs, deficient ossification in the skull, lumbar vertebrae, extremities, and sacral and ischial bones. However, few cases of this osteodysplasia have been described. ${ }^{3}$

The term "achondrogenesis" was first mentioned by Fraccaro in 1952, while describing a stillborn female with severe micromelia and histologic cartilage changes. ${ }^{3}$ Type IA was first described by Houston and Harris for two siblings with short and fractured ribs, completely unossified spines, arched ilia, and hypoplastic ischia. ${ }^{4}$ Genetic and experimental analysis has shown that ACG1A is caused by a mutation in the thyroid hormone receptor interactor 11 gene (TRIP11) encoding Golgi microtubule-binding protein 210 (GMAP-210) required for efficient glycosylation and cellular transport
Department of Basic Medical Sciences,

Center for Research on Congenital

Anomalies and Rare Diseases (CIACER),

L Building, Universidad Icesi, Cali 76003I,

Colombia

Tel +5725552334 ext 8075

Email hmpachajoa@icesi.edu.co 
of multiple proteins. ${ }^{2}$ Other TRIP 11 mutations could lead to abnormal skin, altered chondrogenesis, and lethal skeletal dysplasias. ${ }^{2,4}$

We report the first Latin American case of ACG1A in a male fetus from Colombia with clinical, radiological, and molecular diagnosis. Whole exome sequencing (WES) was performed on fetal DNA and parental blood, which identified two novel pathogenic variants in TRIP11 gene.

\section{Case presentation}

The fetus described was of the first pregnancy of non-consanguineous, healthy Colombian parents; the mother and father were 26 and 28 years old, respectively. The family history was unremarkable. During an apparently normal pregnancy, ultrasound examination results at a gestational age (GA) of 14 weeks revealed fetal nuchal translucency of $4.3 \mathrm{~mm}$, a normal thorax, and short extremities. Ultrasound at GA $20+1$ showed a male fetus with a bell-shaped trunk, narrow thorax, absent stomach pouch, and severe limb shortening. A diagnosis of lethal skeletal dysplasia was suggested. WES of amniotic fluid revealed a diagnosis of ACG1A. The parents received genetic and psychological counseling. However, the mother decided to continue the pregnancy. At GA 28 weeks, the fetus was vaginally delivered with a weight of $1122 \mathrm{~g}$ (49th centile), a height of $28.5 \mathrm{~cm}$ (1st centile), and a head circumference of $25 \mathrm{~cm}$ (19th centile). Following delivery, the fetus manifested shortness of breath and died after a few minutes. Genetic counseling was given to the parents and their families.

Postmortem X-rays and autopsy were performed (Figure 1). Findings showed turricephaly, cerebral gyrus flattening, a hypoplastic nasal bridge, short neck and trunk, short and bowed extremities, club foot, and hypoplastic lungs. The external genitalia were unremarkable. Postmortem radiography showed deficient mineralization of the calvaria and vertebral bodies, unossified sacrum, hypoplastic thorax, and markedly short and beaded ribs with flared and spurred ends. Pathological examination of the fetal sternum and femur tissue with hematoxylin-eosin staining demonstrated hypercellularity of bone and myxoid cartilage matrix; cytoplasmic inclusions were noted and described as "vacuolated chondrocytes" (Figure 2).
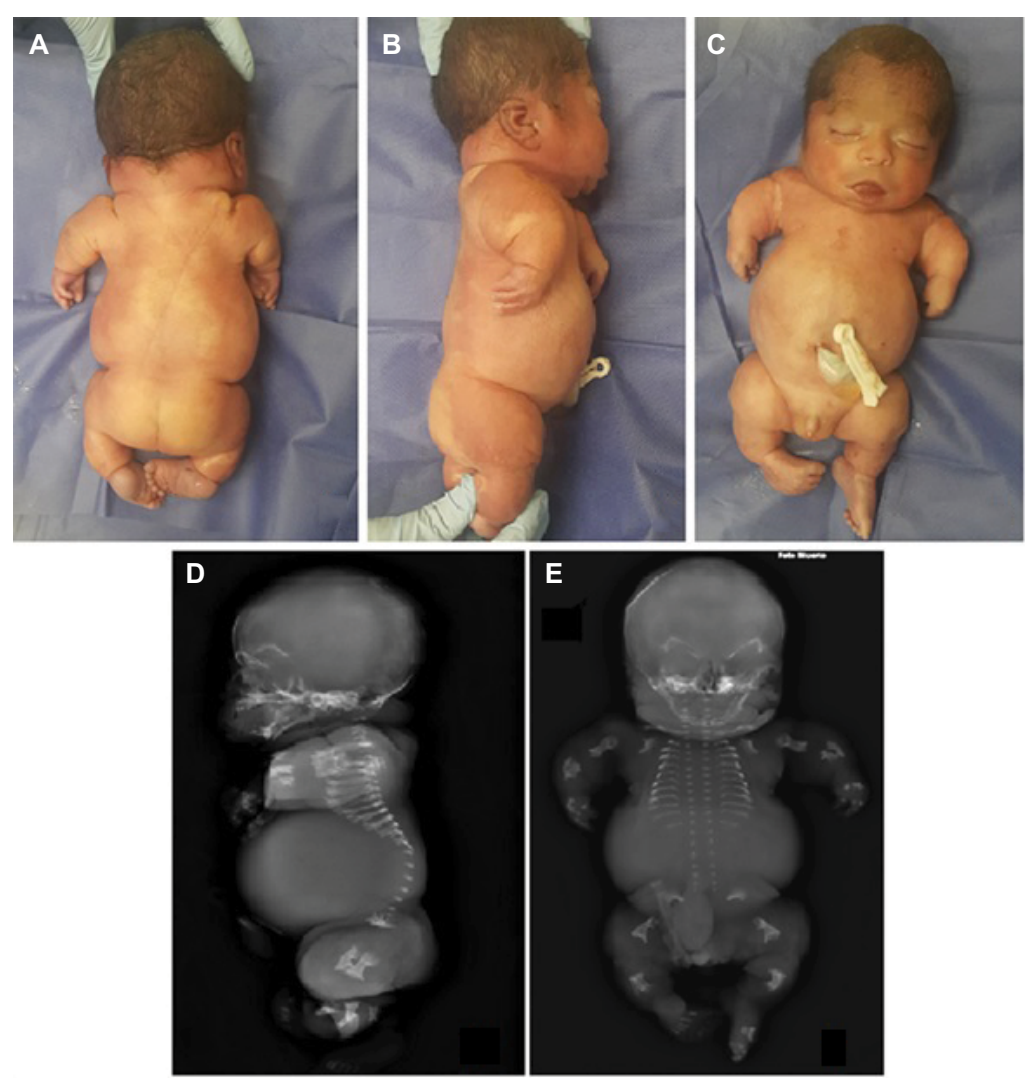

Figure I Patient phenotype and postmortem X-rays.

Notes: (A-C) Posterior, lateral, and frontal views. Patient phenotype shows flat face, short nose, protruding tongue and eyes, low-set ears, narrow bell-shaped thorax, and severe micromelia. (D, E) Postmortem X-ray. Lateral and frontal views showing deficient mineralization of the calvaria and vertebral bodies, unossified sacrum, hypoplastic thorax, and markedly short and beaded ribs with flared and spurred ends. 


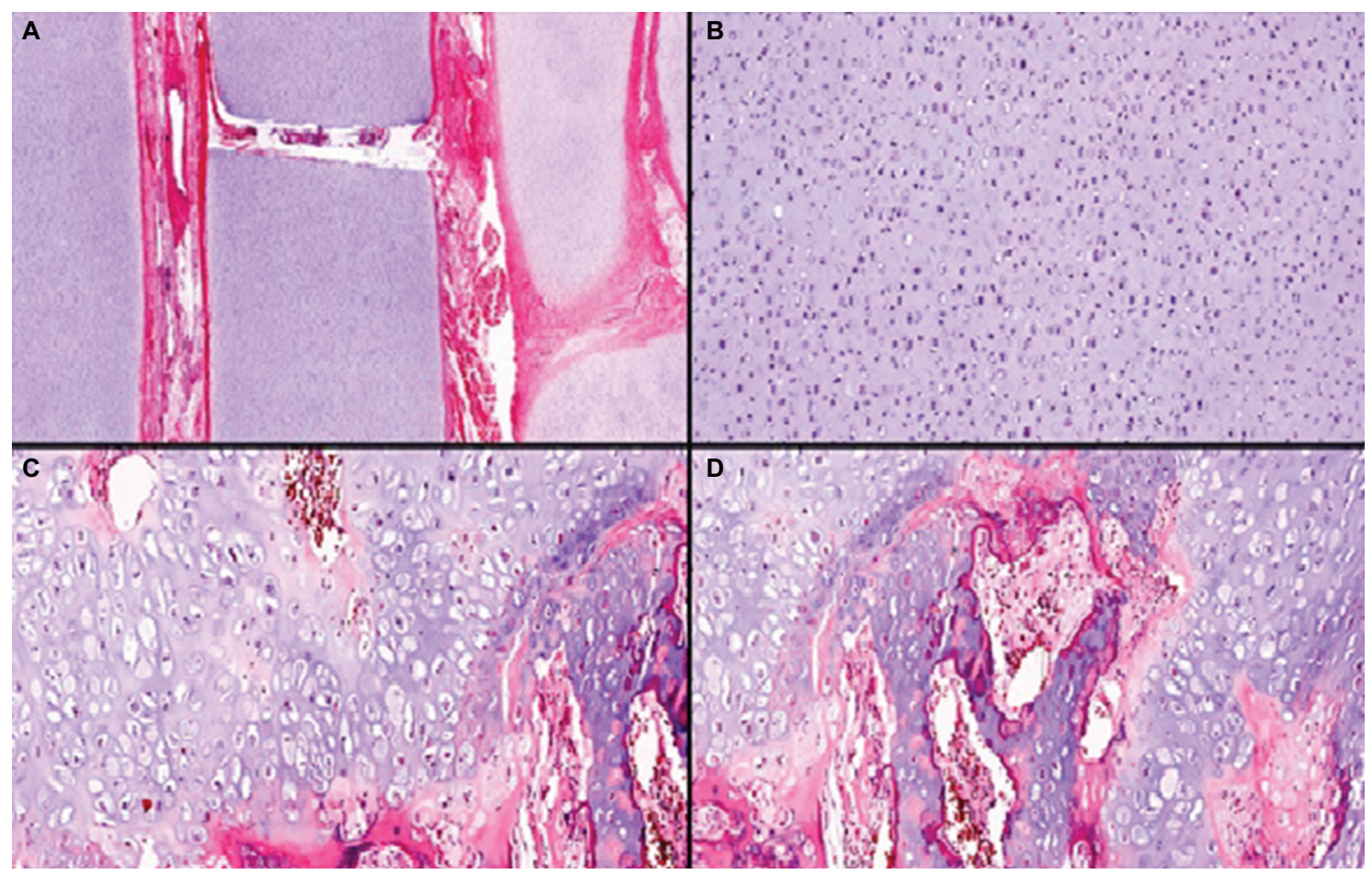

Figure 2 Fetal cartilage section stained with hematoxylin-eosin.

Notes: (A) Costochondral junction region magnified $4 \times$ reveals hypercellularity in the myxoid matrix. The fibrillary matrix tends to form rings around chondrocytes, simulating vacuolated cells. (B) Costochondral junction region magnified 10x reveals hypercellularity in the myxoid matrix. (C) Femoral region magnified 40x reveals hypercellularity in the myxoid matrix. (D) Femoral region magnified $40 \times$ reveals vacuolated chondrocytes.

\section{Materials and methods}

WES was performed on fetal DNA and parental blood. Coding and flanking intron regions were enriched using Agilent in-solution technology and sequenced using the Illumina HiSeq 2500/4000 system. Coverage of at least 30 high-quality sequencing reads per base was achieved by high-throughput sequencing. Only variants in coding and flanking intron regions ( $\pm 8 \mathrm{bp}$ ) with a minor allele frequency (MAF) of $1.5 \%$ were evaluated. Known disease-causing variants (according to the Human Gene Mutation Database) were evaluated up to \pm 30 bp flanking regions and 5\% MAF. The following databases were used for MAFs: 1000 Genomes, dbSNP, Exome Variant Server, and Exome Aggregation Consortium. These variants have not been previously reported in the literature, and the finding was confirmed by Sanger sequencing (Figure 3).

\section{Ethics and consent}

Written informed consent was obtained from the patient's parents for the publication of the case details and accompanying images. Data were collected in the context of studies performed in accordance with the Declaration of Helsinki Good Clinical Guidelines and protocol \#509 "registry of surveillance and survival congenital defects of the Colombian
South-West", approved by the Ethics Committee of Universidad Icesi (Act 192/2011).

\section{Genetic results}

WES identified two fetal TRIP11 heterozygous variants, c.2304_2307delTCAA (p.Asn768Lysfs*7) and c.2128_2129delAT (p.lle710Cysfs*19), inherited from the mother and the father, respectively. Both variants cause a premature stop codon resulting in nonsense-mediated decay of mRNA, leading to loss of protein function. Variant functional prediction software tools (PolyPhen-2, Condel, and SIFT) classified the variants as deleterious. No additional variants were identified, including the genes associated with other skeletal dysplasias, such as SCL26A2 related to achondrogenesis type IB.

\section{Discussion}

Constitutional disorders of the bone are classified as dysostoses, which are malformations of single or multiple bones in combination due to alterations in blastogenesis that cause bone defects and skeletal dysplasias (osteochondrodysplasias) affecting normal bone development or cartilage growth and texture due to genetic mutations. Their resultant pheno- 


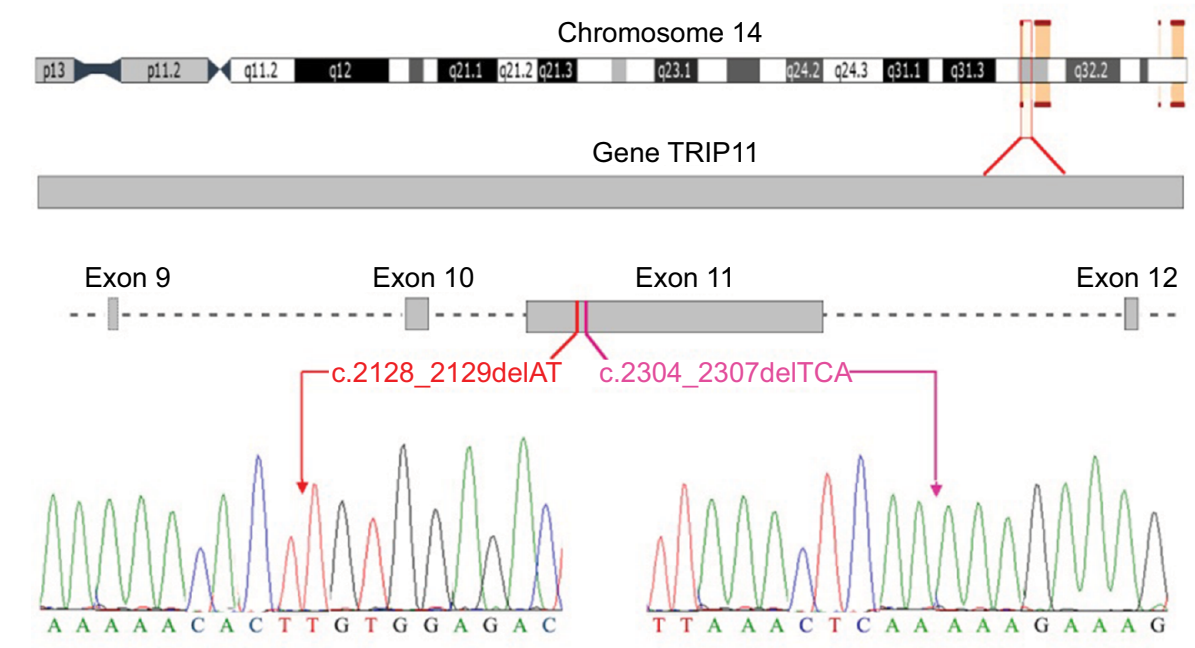

Figure 3 Exon II Sanger sequencing of TRIPII gene showing the deletion of AT (red arrow) at position 2128-2I29 (c.2128_2I29delAT) and deletion of TCA at position 2304-2307 (c.2304_2307) which has an effect on the protein and generates a premature stop codon at amino acids 710 and 768, respectively.

types show lifelong evolution; ${ }^{5}$ consequently, multiple bones of the axial and appendicular skeleton, as well as bones that undergo intramembranous and endochondral ossification, are usually involved. ${ }^{6}$

Around 1970, two types of achondrogenesis, type IA and type IB (ACG1B), were defined using a combination of clinical, radiological, and histologic criteria. These conditions described individuals with similar clinical features: hydrops fetalis, short neck and trunk, narrow thorax, protuberant abdomen, and severe micromyelia. Type IA was first described by Houston and Harris, and later classified histologically by Borochowitz et al. ${ }^{3}$ Distinguishing between ACG1A and ACG1B on clinical and radiological grounds is difficult. However, radiographic findings are slightly different. Fetuses with ACG1B have been described with mildly low ossified areas of the skull, ossified vertebral lateral pedicles, short ribs with no fractures, short femurs with trapezoid configuration, and stellate tibias and humerus. Histopathologic observations show coarse and denser collagen fibers around chondrocytes, giving a "collagen ring" appearance. ${ }^{6}$

Radiological signs in ACG1A include absence of endochondral ossification of the skull and vertebral bodies, short horizontal ribs, flared ribs with multiple fractures, and extremely short tubular bones with most marked changes found in the femur, radius, and ulna. Histologic findings are characterized by hypercellularity in the resting zone, and vacuolated and slightly enlarged chondrocytes with unusual cytoplasmic inclusion bodies denoted as "bull eye cells".,3 Proliferative cartilage lacks cell column formation. These ACG1A characteristics are similar to those in our case (Figure 1). ACG1A histochemical analysis by authors ${ }^{4,6}$ revealed chondrocytes with signs of intracellular retention of proteinaceous material and dilation of the rough endoplasmic reticulum. Subsequently, Aigner et al proposed that amorphous material accumulation causes disturbances in cartilage matrix assembly, affecting chondrocyte differentiation and resulting in disturbances in endochondral bone formation. ${ }^{2}$

The genetic basis of ACG1A was established by analyzing mutant mice with loss of function of TRIP11. ${ }^{1}$ Affected mice died from skeletal dysplasia that shared phenotypic features with ACG1A patients. TRIP11 encodes GMAP-210, which is involved in microtubule anchoring and membrane fusion activities, and also contributes to the assembly and maintenance of the Golgi ribbon around the centrosome. ${ }^{7}$ Therefore, the absence of this protein alters the Golgi structure and maintenance in tissues containing fibroblasts, osteoblasts, and chondrocytes. Descriptions of patients with ACG1 have identified accumulative proteinaceous material and swelling of the rough endoplasmic reticulum in chondrocytes, causing impaired differentiation and apoptosis of these cells. ${ }^{2}$

\section{Conclusion}

To our knowledge, this report is the first case of ACG1A in South America with a clinical, radiological, and molecular diagnosis. ${ }^{4}$ Prenatal WES demonstrated two novel heterozygous frameshift variants in TRIP11 (c.2304_2307delTCA) and (c.2128_2129delAT) that may correlate with a severe phenotype of this condition.

This report supports accumulating evidence of the significant role of TRIP11 in chondrocyte regulation and survival. Additionally, clinical and molecular diagnosis of skeletal dysplasias in utero is essential for genotype-phenotype 
correlation and is useful for providing appropriate prenatal diagnosis and genetic counseling.

\section{Acknowledgments}

We would like to thank the patient's family for their participation in this study. The authors have indicated they have no financial relationships relevant to this article to disclose and no external funding was used.

\section{Author contributions}

All authors contributed toward data analysis, drafting and revising the paper and agree to be accountable for all aspects of the work.

\section{Disclosure}

The authors report no conflicts of interest in this work.

\section{References}

1. Smits P, Bolton AD, Funari V, et al. Lethal skeletal dysplasia in mice and humans lacking the golgin GMAP-210. N Engl J Med. 2010;362(3): 206-216

2. Aigner T, Rau T, Niederhagen M, et al. Achondrogenesis Type IA (Houston-Harris): a still-unresolved molecular phenotype. Pediatr Dev Pathol. 2007;10(4):328-334.

3. Borochowitz Z, Lachman R, Adomian GE, Spear G, Jones K, Rimoin DL. Achondrogenesis type I: delineation of further heterogeneity and identification of two distinct subgroups. J Pediatr. 1988;112(1):23-31.

4. Harris R, Patton JT, Barson AJ. Pseudo-achondrogenesis with fractures. Clin Genet. 1972;3(6):435-441.

5. Panda A, Gamanagatti S, Jana M, Gupta AK. Skeletal dysplasias: a radiographic approach and review of common non-lethal skeletal dysplasias. World J Radiol. 2014;6(10):808-825.

6. Yang SS, Heidelberger KP, Bernstein J. Intracytoplasmic inclusion bodies in the chondrocytes of type I lethal achondrogenesis. Hum Pathol. 1976;7(6):667-673.

7. Wong M, Munro S. Membrane trafficking. The specificity of vesicle traffic to the Golgi is encoded in the golgin coiled-coil proteins. Science. 2014;346(6209):1256898.
The Application of Clinical Genetics

\section{Publish your work in this journal}

The Application of Clinical Genetics is an international, peer-reviewed open access journal that welcomes laboratory and clinical findings in the field of human genetics. Specific topics include: Population genetics: Functional genetics; Natural history of genetic disease; Management of genetic disease; Mechanisms of genetic disease; Counselling and ethical

\section{Dovepress}

issues; Animal models; Pharmacogenetics; Prenatal diagnosis; Dysmorphology. The manuscript management system is completely online and includes a very quick and fair peer-review system, which is all easy to use. Visit http://www.dovepress.com/testimonials.php to read real quotes from published authors.

Submit your manuscript here: https://www.dovepress.com/the-application-of-clinical-genetics-journal 